\title{
Trends in Free Sugar Content of Slovenian Pre-Packaged Foods and Non-Alcoholic Beverages
}

\author{
Nina Zupanic and Igor Pravst \\ Nutrition Institute, Ljubljana, Slovenia
}

\section{Abstract}

In 2015, World Health Organization (WHO) has issued guidelines to reduce the consumption of free sugars to no more than $10 \%$ of the total daily energy intake, with additional health benefits achieved when aiming to less than 5\%. For the general population, following these recommendations has proven difficult due to the massive amount of free sugar available in pre-packed products on the market. In Slovenia, a series of actions have been undertaken to reduce the availability and consumption of foods high in free sugar, including food industry responsibility pledges from soft drink and dairy industry (in 2015 and 2017, respectively).

To monitor the efficacy of those actions as well as general trends in free sugar content on the Slovenian food market, the crosssectional study from 2015 was repeated in 2017. Data from 21,115 pre-packed food items were systematically collected from major retailer shops in Ljubljana, Slovenia. All products were photographed and their European/International Article (EAN) codes scanned to assemble an online database. The products were later assigned to one of the 49 pre-defined food categories, matching those from 2015. The categories that contributed to free sugar consumption in 2015 the most were re-analysed in 2017.

Results showed that in the category of Chocolate and sweets, which in 2015 contributed one third of all free sugar sold on Slovenian market, mean free sugar content increased by $4.7 \%$. Among Soft drinks, which followed shortly after, free sugar content dropped by 8 $\%$. The decrease was also observed among Jellies (10.7\%) Yogurt products $(5.7 \%)$, Breakfast cereals $(1.7 \%)$, Biscuits $(0.9 \%)$, as well as Fruit and vegetable juices $(0.9 \%)$. On the other hand, large increase was observed among Ice creams and edible ices (31.3\%) and in the category of Jam and spreads, in which mean free sugar content increased by $20.3 \%$.

The data showed some favourable trends in free sugar content in many food categories that contribute an important share to an overall free sugar consumption. However, free sugar in certain food categories such as Chocolate and sweets is still on the rise, exposing the need for additional actions that would encourage industry to reformulate products with a lesser amount of free sugar. Moreover, industry self-regulation may be one of, but not the only measure to efficiently reduce free sugar consumption among general population.

\section{Conflict of Interest}

There is no conflict of interest. 